\title{
Haptic Playback: Modeling, Controller Design, and Stability Analysis
}

\author{
Matteo Corno and Miloš Žefran \\ Department of Electrical and Computer Engineering \\ University of Illinois at Chicago \\ Chicago, IL 60607 \\ Email: mzefran@uic.edu
}

\begin{abstract}
Teaching of sensorimotor skills is often considered one of the most promising applications of haptics. Surgical training and rehabilitation are just some of the areas where such training could have large impact. In many cases, the skill to be taught involves forces that have to be exerted along well defined motion trajectories. However, traditional haptics is not capable of displaying both these modalities simultaneously. This paper proposes a novel engineering analysis of haptic playback, a paradigm that allows to simultaneously display force and position data to a user. The analysis is based on treating the human operator as a multiple-input single-output (MISO) system, where the impact of the visual information through which the position data is displayed is explicitly modeled. An intuitive and simple model for the operator is proposed along with a preliminary validation through studies of human subjects. The model is then used to design a novel control strategy that achieves simultaneous display of force and position data. Subsequently, we present the control-theoretic analysis of the proposed approach and results of experiments with human subjects.
\end{abstract}

\section{INTRODUCTION}

Haptics is generally considered to be an enabling technology for teaching of sensorimotor skills. One of the primary targets of the research community has been surgical training, where new laparoscopic, endoscopic, and arthroscopic technologies require constant updating of the practitioner's skills [1] [7]. Traditional media such as textbooks and videotapes are not suitable for teaching motor skills, they are best taught by an instructor through physical interaction. However, such approach is expensive and inefficient because it necessarily requires a low trainee-to-trainer ratio.

Haptic systems have been considered as an alternative to the time-consuming and expensive process of teaching by a human instructor, especially since they can implement additional features such as the ability to record and evaluate the progress of a student. Traditionally, haptic systems are simulators that allow users to explore a virtual environment [8], [9]. In contrast, specialized motor skills used by for example surgeons, artists, or repair technicians often require precise coordination of motion with the force exerted during the motion. For training of such skills, as well as for physical rehabilitation where the coordination of motion and effort is also required, the traditional haptic simulators are clearly inappropriate.

Another deficiency of haptic simulators is that they rely on virtual models of the world. Good mathematical models, especially of biological tissues, are rare and if available they are computationally demanding [10].
These considerations motivated the development of an alternative paradigm for applying haptics to teaching of sensorimotor skills that require a fine and precise interaction with the environment through a tool that contacts the environment at a single point. We base our work on ideas described in [11], [12], [13], and [14] and call our approach haptic playback ${ }^{1}$ since the system should display (enable the user to follow) pre-recorded force and position trajectories. The focus of this paper is a formal model for the haptic playback and rigorous control-theoretic analysis. As part of this process we also develop a new model for human operator and show that the model is necessary for the stability analysis.

\section{A. Motivation}

Most of the existing work in haptics is based on the twoport framework as described for example in [15], [16]. In this framework the haptic system is represented as a network composed of three different elements: the human operator, the haptic interface and the virtual environment [15]. The stability results obtained within this framework rely on the passivity assumption. If the user is assumed to be passive, as suggested by [17], then [18] shows that it is possible to find conditions that guarantee the absence of oscillations for a broad set of nonlinear virtual environments, both delayed and non-delayed.

However using haptics to teach sensorimotor skills that require force and motion coordination is fundamentally different from using a haptic simulator to create a virtual environment in which the user can move and haptically interact with. For the former, the motion of the user in the haptic environment and the force response are constrained by the task, while for the latter the user can freely move in the environment and discover shapes, textures, or dynamic properties of the objects.

In this work we consider fine sensorimotor tasks where the user interacts with the environment through a tool. Such tasks can be described by a pair of trajectories: the trajectory of the position of the contact point and the trajectory of the force exerted at the contact point. In order to successfully complete an interaction task the trainee must be able to follow the position trajectory while exerting the right force along the trajectory. The best way to teach such sensorimotor skills is therefore to display both the position and the force trajectories simultaneously, thus the need for haptic playback. Given

\footnotetext{
${ }^{1}$ The term "playback" is used in the same way as in audio or video playback.
} 
the position (force) trajectory, the force (position) trajectory could be computed from a model of the virtual environment. Alternatively, both trajectories can be recorded while a task is performed by an expert. In this case, haptic playback is analogous to recording a video of a teacher performing the task and then playing the video to the students as many times as needed.

\section{B. Related Work}

Teaching sensorimotor skills through haptics, or more generally through force feedback, has a long history. An excellent analysis of the issues involved is presented in [19]. Recent studies on the role of force feedback in skill acquisition are [20] and [21]. Alternatively, position guidance with haptics is often implemented through virtual fixtures (see [22], [23] for early work and [24] for a recent application). Force feedback is also used extensively for teaching of sensorimotor skills in the rehabilitation robotics literature. Similarly to approach we take, several authors formalize the teaching problem as a control problem so that control theory tools can be used [25], [26].

The idea of simultaneously displaying force and position data has been used before. For example, [11] proposes several schemes to achieve that. Since it is impossible to haptically display both the force and position information at the same time with one haptic device many authors have focused on haptically displaying one of the two trajectories and providing information on the other through a different modality. For haptic systems, the most natural modality is obviously a visual representation. Several attempts have been made in this direction. For example, [12] develops the Virtual calligraphy system to teach calligraphy, [13] describes Virtual haptic back to teach palpation, and [14] the Fingertip presser to simulate pressing on a surface while moving. Worth mentioning is also the first commercial realization of a robotic exercise machine patented by Book [27].

Although these works recognize the need for an additional modality since force and position data can not be displayed simultaneously with a haptic device, the described systems are primarily experimental and do not present any formal justification for or analysis of their approach. The results are thus limited to their particular devices and applications. In contrast, our work, while using some of the same ideas, develops for the first time a formal model for haptic playback. Using the model, various implementations of the haptic playback can be derived and formally evaluated. As a result, the proposed framework is largely independent of a particular system or application.

\section{A Formal Model of Haptic Playback}

As discussed above, the traditional two-port framework is not adequate to study haptic playback since it lacks the ability to properly present to the user the force and position trajectories describing a particular sensorimotor skill. However, causality prevents these two information modalities to be simultaneously displayed with a haptic device. We are thus proposing a new framework in which haptic playback can be properly described. This new framework allows the haptic playback problem to be formulated as a control problem.

The main idea is that haptics is used to display one of the two trajectories while an additional modality is used to display the other. This additional modality, indeed present in several previous applications but never explicitly modeled, overcomes the causality limitations of haptics and thus makes it possible to consider teaching applications. In our work, the additional modality is a visual target overlaid over the virtual environment displayed on the graphical display.

While most haptic simulations use graphics display, visual information is not considered in the two-port framework. In particular, if the human operator is modeled purely as impedance or admittance, the visual feedback is not used by the user. This is in stark contrast to haptic playback where the visual target moving on the graphics display plays a critical role. It is worth pointing out that the active role that the visual feedback plays in haptic playback also distinguishes our our work from [28] and [29].

To formally describe haptic playback, we need to make two assumptions:

1) The system is equipped with a visual representation of the environment, that has at least two elements: the current position of the user (virtual proxy) and a target.

2) The user is cooperative, meaning that she will try to follow the target displayed on screen at the best of her capabilities.

The assumption of having a cooperative user is typical in haptics, while the first assumption has to do with the implementation of the haptic playback.

The framework we propose is schematically represented in Fig. 1. The main difference from the two-port framework is the human operator, called user in figure. The user is not modeled as a two-port element. It is now considered to be a dynamical system with three inputs and one output. The inputs are:

- $\mathbf{x}_{\mathbf{0}}$ is the trajectory of the target displayed on the screen. It can be modulated by the control strategy.

- $f_{\text {act }}$ is the force that is currently exerted by the haptic device. This force is defined by the control algorithm.

- $\mathbf{x}_{\mathbf{u}}$ is the position of the manipulandum.

At the haptic level, the user has the causality structure of an impedance [15]: it accepts a flow and yields an effort. The user has only one output, $f_{u}$ which is a force. In the above figure the block named haptic display represents the haptic device itself and the control logic - for clarity the two parts have been kept together. The system can be seen as a mechanical system with two external forces, $f_{u}$ and $f_{a c t}$. A simple 1 DOF haptic display can be for example modeled as

$$
m \ddot{x}_{u}=f_{a c t}+f_{u}
$$

This framework allows us to study the issues involved in haptic playback from a control theory standpoint. It also has two important implications: (1) it is not possible to study the system with network theory tools and (2) the results of passivity are not directly applicable. Given that network theory and passivity are not applicable, more general tools such as 


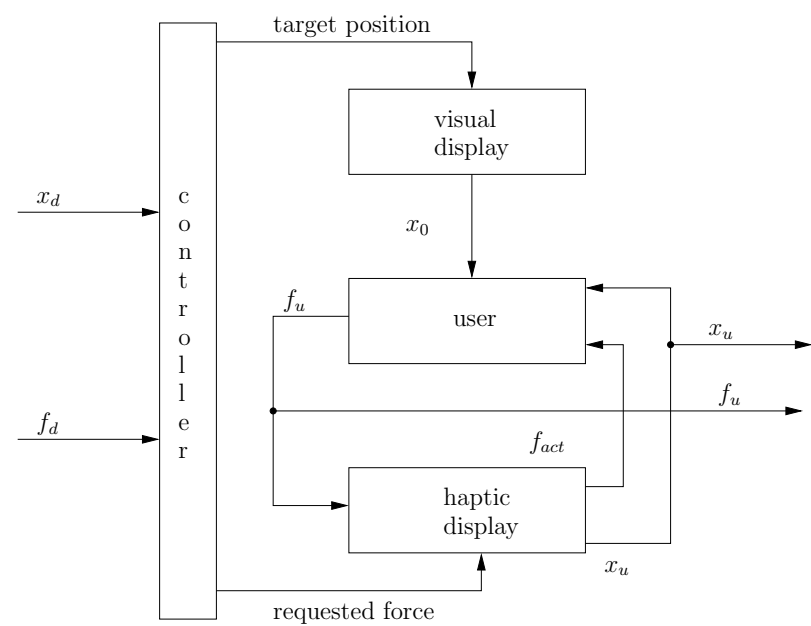

Fig. 1. Proposed framework for haptic playback. The visual channel is explicitly represented.

Lyapunov theory [30] have to be used for stability analysis. Therefore an explicit model of the user is needed.

\section{USER MODEL}

Many researchers in different fields attempted to model human motor dynamics [31]-[37]. Several studies have been aimed at rehabilitation and some were used in haptic stability analysis. It is obviously very difficult to write an accurate model of the human. In most cases accurate models can be obtained only under very constrained and controlled experimental settings, so they can be applied only when those conditions are met. The approach we use to develop the model is inspired by [36] and specifically tailored to haptic playback applications. It is based on some assumptions on the central nervous system (CNS), but we do not claim it to be a rigorous model from the cognitive science point of view. The problem we address is how the user's CNS "computes" the force to be requested from the muscles. We need the model to design control laws for haptic playback, our goal is not to explain how human motor control functions. The choice of an impedance-like causality follows the traditional choice of causality adopted in literature for low inertia and backdrivable haptic devices [38]-[40]. Under assumptions (1), (2) and from a control engineering standpoint, the user is seen as a controller that evolved to minimize the discrepancy between the actual position and the position of the target. The controller consists of two terms, a closed loop on the position error $x_{u}(t)-x_{0}(t)$ and a feedforward term based on the external force which models the adaptation mechanism hypothesized by Mussa-Ivaldi. The resulting transfer function can be written as:

$$
F_{u}(s)=-k e^{-s \tau}\left(X_{u}(s)-X_{0}(s)\right)+(\alpha s-1) F_{a c t}(s) .
$$

The model depends on five parameters. In the position feedback term, $k$ describes a stiffness and $\tau$ the inevitable delay involved in the hand-eye coordination [41]. The other parameters describe the adaptation term. The underlying idea is that the user tries to move toward the displayed target matching the displayed force and adding a force which is proportional to the position error. The term $\alpha s F_{a c t}(s)$ accounts for an error in matching the displayed force. It is ideally modeled as a PD term. It is reasonable to assume that if $f_{\text {act }}$ changes slowly the user will be able to match the displayed force more easily, so the error is proportional to the rate of change of $f_{\text {act }}$. To make the model proper, the differential term is approximated by $\alpha \frac{s+\bar{z}}{s+\bar{p}} F_{a c t}(s)$, yielding

$$
F_{u}(s)=-k e^{-s \tau}\left(X_{u}(s)-X_{0}(s)\right)+\left(\alpha \frac{s+\bar{z}}{s+\bar{p}}-1\right) F_{a c t}(s) .
$$

where $z_{b a r}$ and $p_{b a r}$ are the zero and pole of the first order filter approximating the derivative term, respectively. It is furthermore arbitrarily assumed that the force exerted in each direction is independent from the other directions, so that we can focus on a 1 DOF problem.

\section{USER VALIDATION}

\section{A. Experimental Setup}

To test the user model described by Eq. 3 we used an experimental setup consisting of a Sensable PHANToM ${ }^{\mathrm{TM}}$ PREMIUM 1.0 [42] haptic device interfaced with a PC running Windows 2000. The personal computer was an Intel Xeon $1500 \mathrm{Mhz}$ with $1 \mathrm{~GB}$ of RAM. The PHANToM ${ }^{\mathrm{TM}}$ was equipped with the optional gimbal encoders and the manipulandum, the actual man-machine point of contact, was a stylus that resembles a pen.

\section{B. Human Subjects}

In order to validate the model and estimate the five parameters, 4 subjects were recruited. The subjects were chosen among right handed males with no known history of neurological disorder and ranging in age from 23 to 28 . All the subjects were familiar with the concept of haptic simulation and they all had the chance to experiment with a haptic simulation but had no previous experience with haptic playback.

\section{Experimental Procedure}

The subjects were told to sit in front of the screen and asked to make themselves comfortable. They were told to hold the stylus and instructed to follow a target clearly marked on the screen. As a consequence, each subject was free to assume any posture he found comfortable. It is recognized that this choice affects the repeatability of the experiments, but it also does not limit the results to a very controlled setting as in e.g. [36]. The haptic device was placed so that the $y$ axis of the workspace reference frame was vertical, pointing upward, the $z$ axis horizontally pointed toward the user and the $x$ axis pointed to the right of the user. The target was initially fixed with no force displayed. After a randomly chosen period of time $\bar{t}$ the target was moved to the new position $\bar{x}$ and a constant force $\bar{f}$ displayed. Both values were randomly chosen along the $x$ direction in order to simplify the analysis of the results. Each subject had two consecutive trials. The results used in the identification and validation of the model were taken from the second trial. 


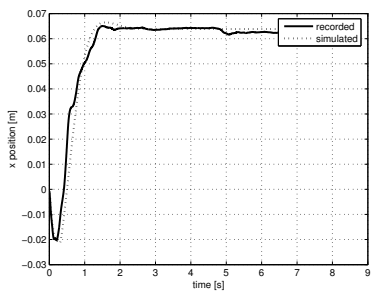

(a) Subject 1 .

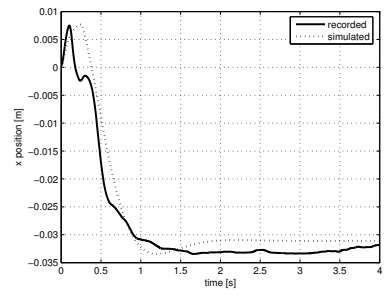

(b) Subject 2

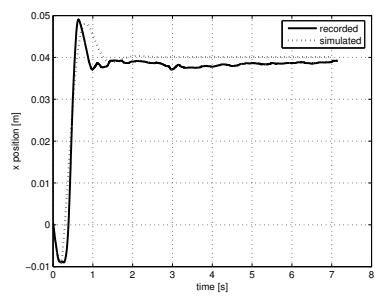

(c) Subject 3 .

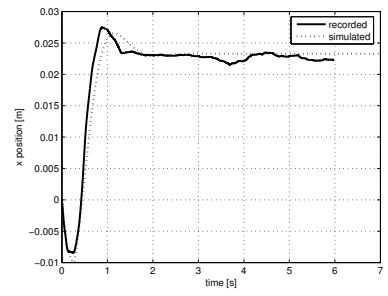

(d) Subject 4 .

Fig. 2. Validation results: simulated and recorded position trajectories.

\section{Validation Results}

The results of the identification experiments are reported in Fig. 2. Each test shows the recorded trajectory and the simulated one. While it is not always possible to get a good matching, in all the cases the time constant, the delay and the overshoot of the response were replicated by the model. This is an indication that the proposed model is able to capture the main features of the user.

\section{Control Strategies}

The discussed MISO framework opens a new possibility. While [12]-[14] recognize the importance of the visual channel in haptic playback, they did not exploit its full potential. In their applications the visual feedback is used in an open loop fashion, it displays the desired trajectory that the user is supposed to learn. The advantages of having a model that describes how the user reacts to changes in $x_{0}$ become clearer if we formulate the problem as a classical control problem: $x_{u}$ and $f_{u}$ are the controlled variables that should follow the references $x_{d}$ and $f_{d}$, while $x_{0}$ and $f_{a c t}$ are the control variables. It is clear that a solution that uses a closed loop control only for $f_{\text {act }}$ is limited with respect to a control architecture that utilizes two closed loops. That is what is a achieved by the novel control strategy we call crossed control. The idea is to use a closed loop strategy to set the target, $x_{0}$, and can be formally described as:

$$
\begin{aligned}
x_{0}(t) & =x_{d}(t)-K_{P}\left(f_{u}(t)-f_{d}(t)\right) \\
f_{a c t}(t) & =-f_{d}(t)-K_{F}\left(x(t)-x_{d}(t)\right)
\end{aligned}
$$

with $K_{P}>0$ and $K_{F}>0$. Intuitively, the target is used to correct the force error, while the force exerted by the device corrects the position error. As a consequence of this strategy the haptic device will drive the user along the correct trajectory letting the user actively exert the force.

To further explain the idea, consider the example of a hard surface penetration where $x_{d}(t)=$ const. while $f_{d}(t)$ is linearly increasing. If the user can perfectly execute the task then the controller will keep the target at the desired position and exert the desired force. If the user exerts less force than requested, the controller will move the target so that the resulting position error causes the haptic device to apply a different force, triggering in turn a transient that, under conditions to be specified later, converges to the desired position and force.

Alternatively, we could use the control strategy adopted by [12] that we will call a direct force controller. It can be described by the following laws:

$$
\begin{aligned}
x_{0}(t)= & x_{d}(t) \\
f_{a c t}(t)= & -f_{d}(t)+K_{P}\left(f_{u}(t)-f_{d}(t)\right)+ \\
& \left(+K_{I} \int_{0}^{t} f_{u}(\xi)-f_{d}(\xi) d \xi\right),
\end{aligned}
$$

where $K_{P}>0$ is the proportional gain, and $K_{I}>0$ is the gain of the optional integral term that is used to avoid steady state error. The force $f_{u}$ is controlled with a PI controller. The target follows the desired trajectory and the controller basically exerts a force which is opposite to the desired one, but adjusted to reduce errors.

The difference between the crossed controller and the direct force controller (and other traditional controllers) is that the former uses two closed loops instead of one and that in the direct force control $f_{u}$ is controlled through reaction, meaning that the exact $f_{u}$ is obtained by exerting the force that was applied when the task was recorded. This can be seen as a passive way of exerting the force because it is based on the resistance that the user offers. It conveys the correct haptic feeling to the user, but psychologically the user does not feel to be the cause of this force, she has to rely on an external force stimulus. We believe that this negatively affects the learning process. In contrast, with the crossed controller the user actively exerts the force; psychologically she is the cause of the force and therefore conceptually the teaching experience is more effective.

\section{Vi. Simulation Results}

Before implementing the direct force controller and the crossed controller on the PHANToM ${ }^{\mathrm{TM}}$, they were tested using the proposed user model. In particular, a system composed of a 1 DOF linear damped mass coupled to the user was used. Two different sets of parameters were tested in simulation. In the first set the user's hand-eye coordination does not introduce any delay; in the second set the user model is delayed according to the identified parameters. Formally, the system used for testing was the following:

$$
m \ddot{x}_{u}+c \dot{x}_{u}=f_{u}+f_{a c t}
$$

with $f_{u}$ determined by the transfer function (3). The numerical values of the parameters used in the simulation were the one identified for the first subject. The crossed controller and the direct controller were simulated too. Initially all the gains were set to 10 and the references $f_{d}$ and $x_{d}$ were subjected to a step at time $t=1 \mathrm{~s}$; a desired position of $0.06 \mathrm{~m}$ was requested 


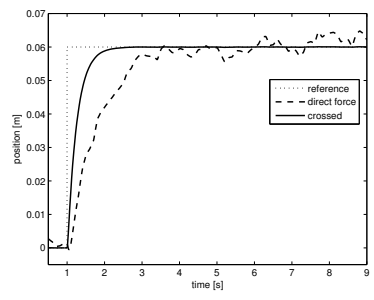

(a) position.

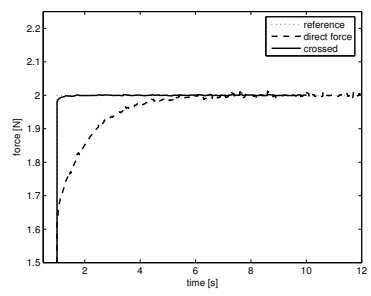

(b) force.

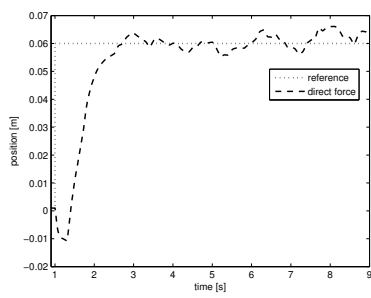

(c) position

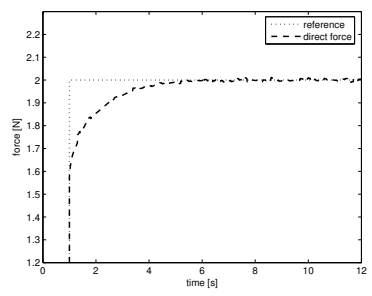

(d) force

Fig. 3. Simulation results, force and position tracking for the non delayed case(a-b) and the delayed case(c-d).

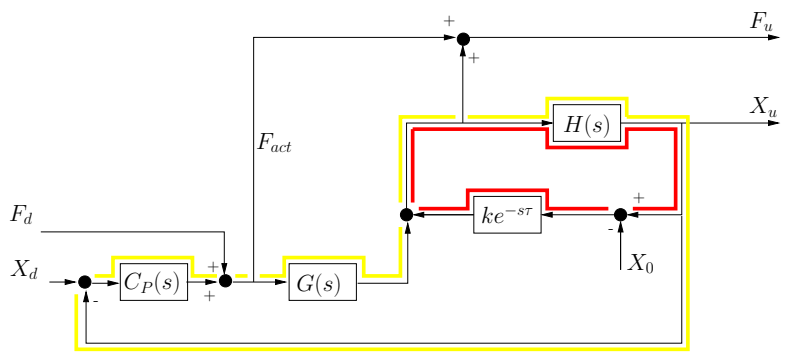

(a) $f_{\text {act }}$ control loop.

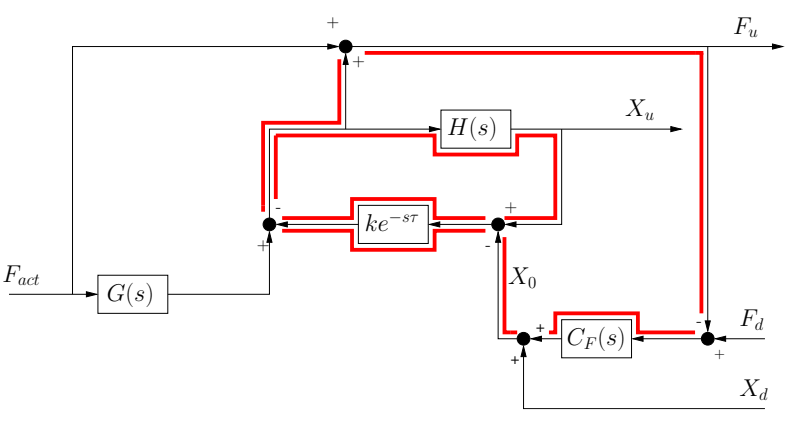

(b) $x_{0}$ control loop.

Fig. 4. Block diagram of the crossed controlled system.

along with a force of $2 \mathrm{~N}$. The results of the non delayed case are shown in Fig. 3.

From Fig. 2 it can be seen that the crossed controller offers better results than the direct force controller not only in terms of tracking but also in noise rejection.

After testing the control laws on an ideal user without delay, the delay was introduced. The results in the delayed case and for the same gains can be seen in Fig. 3(c) and 3(d). The previous observations about the traditional control law still hold, but the outcome for the crossed controller is dramatically different: it is not plotted because the introduction of the delay makes the system unstable. In order to stabilize the system the gain of the position loop must be reduced to $K_{P}<0.25$. According to the simulations the only way to robustly stabilize the system would be to use a small $K_{P}$. This choice would make the crossed controller act like a controller with only one closed loop, loosing its advantage. In order to see whether the crossed controller can be used at all, a more thorough investigation of the problem is needed.

\section{Stability ANALYSIS}

It has been shown that the introduction of the delay in the user model destabilizes the crossed controller, while the same delay affects the performance of the traditional controller only minimally. The main difference between the traditional controller and the crossed controller is an additional closed loop. This is made clear in figures 4(a) and 4(b). The figures show the block diagrams for the two control variables in the crossed controller. Both the traditional direct force controller and the crossed controller have an identical closed loop that computes $F_{a c t}=C_{P}(s)\left(X_{u}-X_{d}\right)$. Fig. 4(a) shows that there are two closed loops: an intrinsic delayed loop (marked in darker gray) which represents the user trying to follow the target, and the loop explicitly closed by the controller $C_{P}(s)$ (marked in lighter gray) which is not delayed. Both the traditional controller and the crossed controller have the intrinsic delayed loop and the non delayed loop used by $C_{P}(s)$. The difference lays in an additional closed loop of the crossed controller, seen in Fig. 4(b). The additional loop is used by the controller $C_{F}(s)$ and it is delayed, an indication that the system can be driven toward instability.

This preliminary analysis suggests that the cause of instability is the delayed loop. Therefore we can simplify the system and focus only on the additional delayed loop. The terms that do not affect stability appreciably are the non delayed loop and the adaptation terms. Using the 1 DOF damped mass all these terms can be rewritten as

$$
m \ddot{x}_{u}(t)+c \dot{x}_{u}(t)=-k\left(x_{u}(t-\tau)-x_{0}(t-\tau)\right)
$$

where

$$
x_{0}(t-\tau)=-f_{d}(t-\tau)-K_{P}\left(f_{u}(t-\tau)-f_{d}(t-\tau)\right)
$$

System (6) can be represented by the block diagram of Fig. 5 . It is useful to start considering the case with no delay, i.e.

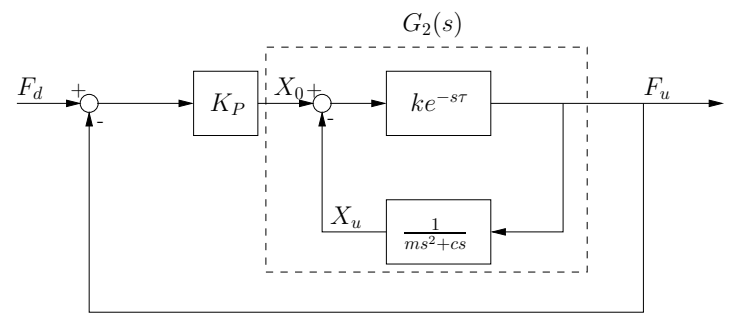

Fig. 5. Block diagram of the simplified model with delay.

$\tau=0$. Recalling that the parameters $m, c, k$ and $K_{P}$ are 
positive, Routh criterion shows that the system is stable for any choice of $K_{P}$.

More problematic is the study of the delayed system. As shown in Fig. 5 we will initially focus on the inner loop transfer function $G_{2}(s)$. Solving the inner loop, we get

$$
G_{2}(s)=\frac{k e^{-s \tau}\left(m s^{2}+c s\right)}{m s^{2}+c s+k e^{-s \tau}}
$$

Solving the outer loop with the loop transfer function $K_{P} G_{2}(s)$ yields the delayed closed loop transfer function,

$$
\begin{aligned}
& H_{f_{d} \rightarrow f_{u}}^{D}(s)= \\
& \quad \frac{K_{P} k e^{-s \tau}\left(m s^{2}+c s\right)\left(m s^{2}+c s+k e^{-s \tau}\right)}{m s^{2}+c s+k e^{-s \tau}+K_{P} k e^{-s \tau} m s^{2}+K_{P} k e^{-s \tau} c s} .
\end{aligned}
$$

It is clear that the transcendental term $k e^{-s \tau}$ makes the system not amenable to classical control tools. One possible way to proceed is to substitute the transcendental term with the second order Padé approximation [43],

$$
e^{-s \tau}=\frac{1-s \tau / 2}{1+s \tau / 2} \triangleq \frac{1-s T}{1+s T} .
$$

Substituting the Padé approximations we can write the denominator of the closed loop transfer function as

$$
\begin{aligned}
D(s) & +K_{P} N(s)= \\
& =m T s^{3}+m s^{2}+c T s^{2}+C s-K T s+K+ \\
& -M T k K_{P} s^{3}+k K_{P} m s^{2}-c T k K_{P} s^{2}+ \\
& +k K_{P} c s
\end{aligned}
$$

The goal is to describe the dependence of the roots of (10) on the two parameters $T$ and $K_{P}$. This can be done using root locus technique [44]. The analysis shows that when $K_{P}<\frac{1}{k}$, the system is stable for small values of $T$ and when $T$ increases eventually there is a value, $T_{\text {crit }}$, that destabilizes the system. When $K_{P}>\frac{1}{k}$, there is always at least one unstable pole, independently of the value of $T$, the system will be unstable. Therefore a necessary condition for the stability of the system is that $K_{P}<\frac{1}{k}$. But this condition is only necessary: to ensure stability $T_{\text {crit }}$ must be found. It is possible to do so using a method derived from the analysis introduced by Olgac in [45]. The method is based on the continuity argument, which states that in the one-dimensional parameter space, $\tau$, there are regions where the number of unstable roots of the transcendental characteristic equation is fixed. In other words the continuity argument states that if $\tau$ is varied continuously, the position of the roots cannot but vary continuously. This analysis results in the following theorem.

Theorem 1: The crossed controller described by

$$
\begin{aligned}
x_{0}(t) & =x_{d}(t)-K_{P}\left(f_{u}(t)-f_{d}(t)\right) \\
f_{a c t}(t) & =-f_{d}(t)-K_{F}\left(x_{u}(t)-x_{d}(t)\right)
\end{aligned}
$$

when coupled to a human user modeled as

$$
F_{u}(s)=-k e^{-s \tau}\left(X_{u}(s)-X_{0}(s)\right)+\left(\alpha \frac{s+\bar{z}}{s+\bar{p}}-1\right) F_{a c t}(s),
$$

is asymptotically stable if and only if

$$
\begin{aligned}
K_{P} & <\frac{1}{k} \\
\tau & <\tau_{\text {crit }}
\end{aligned}
$$

where $\tau_{\text {crit }}$ is the first value of the delay that causes a crossing of the imaginary axis. $\tau_{\text {crit }}$ depends on $K_{P}$.

For example, using the parameters identified for one of the subjects the conditions are found to be $K_{P}<0.25$ and for $K_{P}=0.24$ a limit of $\tau<0.642 \mathrm{~s}$ is computed.

To summarize, in this section some considerations on the stability of the crossed control law have been made; sufficient and necessary conditions for the stability have been found. In particular, a limit for the gain of the loop used to set $x_{0}$ has been found. This limit has been shown to be inversely proportional to the stiffness of the user, and does not depend on the amount of delay. If the gain is above the limit, the system is unstable. If it is below the limit, the system stability will depend on the amount of delay. It is important to note that this conditions have been derived under the assumption of a known user model. Since the model of the user is not perfect, these conditions must be taken as guidelines for designing the controller.

\section{EXPERIMENTAL RESULTS}

In this section the experimental results obtained with the implemented control laws are presented and discussed. The system used for these tests was the same used for the user model validation. With the goal to simplify the task and avoid issues with visualization, only planar tasks were considered. The desired trajectories and forces are generated on the plane $x-y$. The subjects involved in these tests were the same used in the validation experiments.

In the previous section we discussed that with the proposed model of the human user, the crossed controller can go unstable when the gain $K_{P}$ is greater than a threshold which is inversely proportional to the user's stiffness. The first thing that experiments show is that this behavior does not manifest itself. The gain used in tests is well above the expected stability limit, but the trajectories continue to be stable. This behavior can be explained by observing that in the simulation the instability is due to the reference $x_{0}$. The higher the gain is, the more abruptly the target, $x_{0}$, moves. This is true in simulations and in experimental tests as well, but the user model used in simulation is time-invariant. According to the model the user always exerts a force proportional to the error independently of the situation and with a constant stiffness. The more abruptly the target moves the higher the force exerted by the user is. This behavior not only triggers the instability, but sustains it. In a real experiment the user is continuously changing and adapting to the situation. She will never voluntarily inject enough energy in the system to sustain an instability. This is why in real experiments an unstable behavior is rarely observed. Formally, while the user cannot easily influence the condition given by Eq. (12) because it depends on the reaction time, the condition given by Eq. (11) is influenced by the user modulating her stiffness so that when the system moves toward instability stiffness is reduced. 


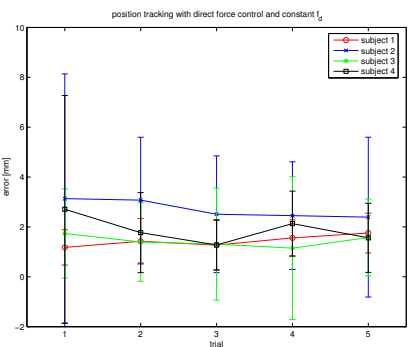

(a) Position error.

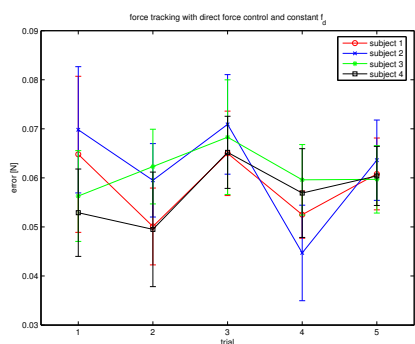

(b) Force error.

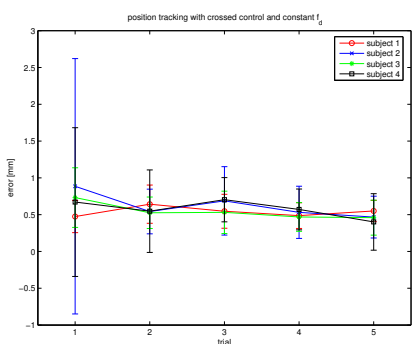

(c) Position error.

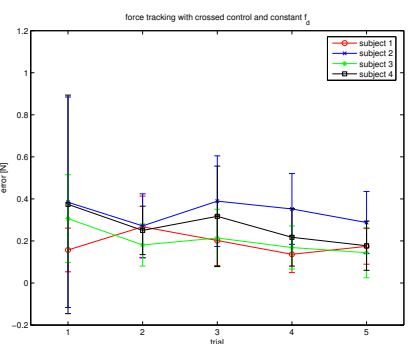

(d) Force error.

Fig. 6. Position and force errors obtained in tests with the direct force controller and the crossed controller (constant force case).

Once the main discrepancy between experimental and simulated results has been studied, a comparison between the two haptic controllers can be carried out bearing in mind that in this phase of our study we focused on the tracking performance of the two strategies, not on their teaching potentials. In order to compare the two strategies the following protocol was designed. The subjects were asked to sit comfortably in front of the system and grab the stylus. No instructions were given on how the stylus should be held. The subjects were asked to follow a target shown on the screen. Four different testing conditions were devised and obtained by combination of two features: the control strategy used and the desired force. The control strategies were the direct force controller and the crossed controller. The desired force followed two trajectories: in the first case the desired force was constant and equal to $f_{d}=[1,0,0]^{T}$, whilst in the second case the desired force was given by

$$
f_{d}=\left[\begin{array}{c}
-0.5-\sin (t) \\
-0.5-\cos (t)
\end{array}\right]
$$

Each subject had five consecutive trials for each condition. Each trial lasted 25 seconds. Between the sets of trials the subject were given the chance to rest for a minute.

Fig. 6 summarizes the results of the tests. Each plot represents the 5 trials for a given testing condition for all the subjects; the average and standard deviation of the position and force errors are given. From the test it was clear that contrary to what is expected, there is no relevant difference between errors when the task is to exert a constant force or when the task is to follow a more complex force trajectory. Therefore we will refer only to the results obtained with constant desired force. The main characteristic that can be observed from these tests is that the direct force controller offers a better force tracking with respect to the crossed controller while the crossed controller offers a better position tracking.

Another expected fact that has been confirmed by these experiments is that with the crossed controller there is a change in the user model that guarantees stability. Fig. 6 shows the first five trials executed by each subject with the crossed controller. It can be seen that the standard deviations of the position errors are great in the first trials and rapidly drop in the subsequent attempts. This is due to the fact that by the end of the first trial all the subjects realized that "the target movement depends on my actions" (as commented by the subjects) and adapt their behavior, thus changing the internal controller they used to follow the target.
Even though the tests were not designed to evaluate teaching capabilities of the two algorithms a certain degree of learning can be detected that manifest as a decreasing trend of the error; this trend does not necessary imply a skill acquisition. Nevertheless an intuitive difference between the two strategies is worth discussing. The crossed controller is believed to be less intrusive. If the user perfectly follows the desired trajectory, $x_{d}$, the haptic device does not exert any force. A force is felt only as a reaction force to a user action. The user, trying to follow the target, $x_{0}$, exerts the force. This does not happen with the direct force controller, the user always feels the force. In the latter case if the user is able to follow the trajectory with no errors, she feels the correct force, but she is not the cause of that force. The correct feeling is due to a reaction to an external force that will not be present when the task is autonomously performed.

\section{COnclusion And Future Work}

We presented a formal framework and analysis of haptic playback, a novel strategy for teaching sensorimotor skills that require precise coordination of motion with the force exerted during the motion. We argued that the stability analysis of haptic playback requires an explicit model of the user. A possible MISO model for the human operator was proposed, validated and discussed. This model is not intended to be a cognitive model for human motor control, it is used solely to capture the main features needed for analysis and design of control laws.

The proposed framework allows a rigorous design and analysis of different control strategies for haptic playback. In particular, we proposed a novel control strategy, crossed controller, and studied its stability. Discrepancies between the results of the stability analysis and the experiments with human subjects have been briefly discussed. The performance of the crossed controller has been evaluated on human subjects and discussed. The results are encouraging and show that a simple model of the user can help designing effective control laws.

While the focus of this study was on the tracking properties of the control laws, in future we plan to study how tracking affects learning. The hypothesis is that when training in traditional haptic simulators is preceded by a phase in which haptic playback is used, the effectiveness of the training depends on the tracking performance of the haptic playback. 


\section{REFERENCES}

[1] N. Ayache, S. Cotin, H. Delingette, J. M. Clemente, Y. Russier, and J. Marescaux, "Simulation of endoscopic surgery," Minimally Invasive Therapy \& Allied Technologies, vol. 7, no. 2, pp. 71-77, 1998.

[2] J. Berkley, G. Turkiyyah, D. Berg, M. Ganter, and S. Weghorst, "Realtime finite element modeling for surgery simulation: An application to virtual suturing," IEEE Transactions on Visualization and Computer Graphics, vol. 10, no. 3, pp. 312-325, 2004.

[3] R. Dayal, P. L. Faries, S. C. Lin, J. Bernheim, S. Hollenbeck, G. DeRubertis, S. Trocciola, J. Rhee, J. McKinsey, N. J. Morrissey, and K. C. Kent, "Computer simulation as a component of catheter-based training," Journal of Vascular Surgery, vol. 40, no. 6, pp. 1112-1117, 2004.

[4] R. Playter and M. Raibert, "A virtual surgery simulator using advanced haptic feedback," Minimally Invasive Therapy, vol. 6, no. 2, 1997.

[5] T. Sohmura, H. Hojo, N. Nakajima, K. Wakabayashi, M. Nagao, S. Iida, T. Kitagawa, M. Kogo, T. Kojima, K. Matsumura, T. Nahamura, and J. Takahashi, "Prototype of simulation of orthognathic surgery using A virtual reality haptic device," International Journal of Oral and Maxillofacial Surgery, vol. 8, no. 33, pp. 740-750, 2004.

[6] R. Sweet, T. Kowalewksi, P. Oppenheimer, S. Weghorst, and R. Satava, "Face, content and construct validity of the university of washington virtual reality transurethral prostate resection trainer," Journal of Urology, vol. 5, no. 172, pp. 1953-1957, 2004.

[7] J. W. Ward, D. P. M. Wills, K. P. Sherman, and A. M. M. A. Mohsen, "The development of an arthroscopic surgical simulator with haptic feedback," Future Generation Computer Systems, vol. 3-4, no. 14, pp. 243-251, 1998.

[8] R. Adams and B. Hannaford, "Control law design for haptic interfaces to virtual reality," IEEE Transactions on Control Systems Technology, vol. 10, no. 1, pp. 3-13, Jan. 2002.

[9] R. B. Gillespie and M. R. Cutkosky, "Stable user-specific haptic rendering of the virtual wall," in Proceedings of the Fifth Annual Symposium on Haptic Interfaces for Virtual Environment and Teleoperator Systems, K. Danai, Ed., vol. DSC 58, International Mechanical Engineering Congress and Exposition. Atlanta, Georgia, USA: Proceedings of the ASME Dynamic Systems and Control Division 1996, Nov. 1996, pp. 397-406.

[10] V. Chial, S. Greenish, and A. M. Okamura, "On the display of haptic recordings for cutting biological tissues," in 10th International Symposium on Haptic Interfaces for Virtual Environment and Teleoperator Systems, vol. 3, 2002, pp. 80-87.

[11] Y. Yokokohji, R. L. Hollis, and T. Kanade, "Sensing and force reflecting exoskeleton (SAFIRE) specification," in Proc. IEEE Virtual Reality Апnu. Int. Symp, 1996, pp. 46-53.

[12] J. Henmi and T. Yoshikawa, "Virtual lesson and its application to virtual calligraphy system." in ICRA, 1998, pp. 1275-1280.

[13] R. L. W. II, M. Srivastava, R. R. C. Jr., and J. N. Howell, "Implementation and evaluation of a haptic playback system," Haptics-e, vol. 3, no. 3, pp. 1-5, 2004.

[14] R. Kikuuwe and T. Yoshikawa, "Haptic display device with fingertip presser for motion/force teaching to human," in Proc. IEEE/CNF Int. Conf. on Robotics and Automation, vol. 1, 2001, pp. 868-873.

[15] R. J. Adams and B. Hannaford, "A two-port framework for the design of unconditionally stable haptic interfaces," in IEEE/RSJ International Conference on Intelligent Robots and Systems, Victoria, B.C., 1998, pp. 1254-9.

[16] B. E. Miller, J. E. Colgate, and R. A. Freeman, "Computational delay and free mode environment design for haptic display," in Proc. ASME Dyn. Syst. Cont. Div., vol. 3, 1999.

[17] N. Hogan, "Multivariable mechanics of the neuromuscular system," Proc. IEEE Annual Conference of Engineering in Medicine and Biology Society, pp. 594-598, 1996.

[18] B. E. Miller, J. E. Colgate, and R. A. Freeman, "Guaranteed stability of haptic systems with nonlinear virtual environments," IEEE Transactions Robotics and Automation, vol. 16, no. 6, pp. 712-719, 2000.

[19] B. Gillespie, S. O'Modhrain, P. Tang, C. Pham, and D. Zaretsky, "The virtual teacher," in Proceedings of the ASME IMECE, vol. 64, Anaheim, CA, 1998, pp. 171-178.

[20] D. Feygin, M. Keehner, and F. Tendick, "Haptic guidance: Experimental evaluation of a haptic training method for a perceptual motor skill," in Proceedings of the 10th Symposium on Haptic Interfaces for Virtual Environment and Teleoperator Systems, vol. 1, 2002, p. 40.

[21] C. Wagner and R. Howe, "Mechanisms of performance enhancement with force feedback," in First Joint EuroHaptics Conference and Symposium on Haptic Interfaces for Virtual Environment and Teleoperator Systems, Pisa, Italy, 2005, pp. 21-29.
[22] J. Funda, T. S. Lindsay, and R. P. Paul, "Teleprogramming: Toward delay-invariant remote manipulation," Presence, vol. 1, no. 1, pp. 2944, 1992.

[23] L. B. Rosenberg, "Virtual fixtures: perceptual tools for telerobotic manipulation," in IEEE Virtual Reality Annual International Symposium (VRAIS'93), Seattle, WA, 1993, pp. 76-82.

[24] J. J. Abbott and A. M. Okamura, "Stable forbidden-region virtual fixtures for bilateral telemanipulation," ASME Journal of Dynamic Systems, Measurement, and Control, vol. 128, no. 1, pp. 53-64, 2006.

[25] J. Patton and F. Mussa-Ivaldi, "Robot-assisted adaptive training: custom force fields for teaching movement patterns," IEEE Transactions on Biomedical Engineering, vol. 51, no. 4, pp. 636-646, 2004.

[26] J. Emken, J. Bobrow, and D. Reinkensmeyer, "Robotic movement training as an optimization problem: designing a controller that assists only as needed," in Rehabilitation Robotics, 2005. ICORR 2005. 9th International Conference on, July 2005, pp. 307-312.

[27] W. Book, D. A. Ruis, and R. W. Polhemus, "Robotic exercise machine and method," U. S. Patent 4,235,437, 1980.

[28] C. Avizzano, M. Raspolli, S. Marcheschi, and M. Bergamasco, "Haptic desktop for office automation and assisted design," in Proceedings of the 2005 IEEE International Conference on Robotics and Automation, Apr. 2005, pp. 4086-4091.

[29] K. Kurihara, F. Suzuki, S.and Harashima, and K. Furuta, "Human adaptive mechatronics (HAM) for haptic system," in 30th Annual Conference of IEEE Industrial Electronics Society (IECON 2004), 2004, pp. 647652.

[30] H. K. Khalil, Nonlinear Systems, 3rd ed. Prentice Hall, 2002.

[31] W. Abend, E. Bizzi, and P. Morasso, "Human arm trajectory formation," Brain, vol. 2, no. 105, 1997

[32] V. B. Brooks, The Neural Basis of Motor Control. Oxford University Press, 1986.

[33] M. H. Raibert, "A model for sensorimotor control and learning," Biological Cybernetics, vol. 29, no. 2, pp. 29-36, 1978.

[34] E. Saltzman and S. J. A. Kelso, "Skilled actions: A task-dynamic approach," Psychological Review, vol. 94, no. 1, pp. 84-106, 1987.

[35] C. J. Hasser and M. R. Cutkosky, "System identification of the human hand grasping a haptic knob," in Proceedings of the 10th Symposium on Haptic Interfaces for Virtual Environment and Teleoperator Systems table of contents, Orlando, 2002, p. 40.

[36] R. Shadmehr and F. A. Mussa-Ivaldi, "Adaptive representation of dynamics during learning of motor task," Journal of Neuroscience, vol. 14, no. 5, pp. 3208-3224, May 1994.

[37] R. E. Kearney and R. F. Kirsch, Neural Control of Posture and Movement. Springer-Verlag, 1999, ch. System identification and neuromuscular modeling, pp. 105-116.

[38] M. Minsky, M. Ouh-Young, O. Steele, F. P. B. Jr., and M. Behensky, "Feeling and seeing: Issues in force display," in Computer Graphics. ACM, 1990, vol. 24, no. 2, pp. 235-243.

[39] E. Colgate, P. Grafing, M. Stanley, and G. Schenkel, "Implementation of stiff virtual walls in force-reflecting interfaces," in Proceedings of VRAIS, Seattle, WA, 1993, pp. 202-208.

[40] S. Salcudean and T. Vlaar, "On the emulation of stiff walls and static friction with a magnetically levitated input/output device," in Dynamics Systems and Control, vol. 55. Chicago, IL: ASME, Nov. 1994, pp. 123130, proceedings of the Third Annual Symposium on Haptic Interfaces for Virtual Environment and Teleoperator Systems.

[41] R. A. Hess, "Unified theory for aircraft handling qualities and adverse aircraft-pilot coupling," Journal of Guidance, Control and Dynamics, vol. 20, no. 6, 1997.

[42] T. Massie and J. K. Salisbury, "The PHANTOM haptic interface: A device for probing virtual objects," in Proc. ASME Winter Annual Meeting: Symposium on Haptic Interfaces for Virtual Environment and Teleoperator System., Chicago, 1994.

[43] J. E. Marshall, Control of Time-Delay Systems. Peter Peregrinus LTD., 1979.

[44] R. C. Dorf and R. H. Bishop, Modern Control Systems, 9th ed. Prentice Hall, 2001.

[45] N. Olgac and R. Sipahi, "An exact method for the stability analysis of time-delayed linear time-invariant systems," IEEE Transactions on Automatic Control, vol. 47, no. 5, pp. 793-874, 2002. 